\title{
Interprofessional Education through a Novel Peer-Assisted Learning Model
}

Interprofessional Education (IPE) may be challenging program components to incorporate into the competency-rich Medical Laboratory Technician (MLT) curriculum. Models of IPE are varied but they all have, at their foundation, two or more professions learning about, from, and with each other to enable effective collaboration to optimize healthcare processes and patient outcomes. Furthermore, studies have demonstrated a strong positive relationship between inclusion of IPE in health education curricula and success in entry-level careers. Incorporating IPE into the curriculum, without loss of other key competencies, requires creativity and necessitates collaboration with content experts from other healthcare fields. This presentation summaries the process and preliminary results of a pilot project involving MLT students as part of an interprofessional team of peer-assisted learning (PAL) tutors, reinforcing physiological processes and clinical diagnostic procedures through laboratory activities in a freshman undergraduate Anatomy and Physiology course. Drawing on this innovative PAL model, as well as other IPE strategies, two new MLT programs in Virginia are implementing learning techniques to meet IPE expectations using collaborative models. In addition, this approach to early IPE strives to promote a culture of interprofessionalism which is introduced in preprofessional courses, reinforced in program curricula, and ultimately mastered throughout the professional career. 